\title{
Powering nuclear security
}

\author{
International collaboration and deep technical understanding are essential to building safe and secure \\ nuclear facilities, particularly where political tensions undermine trust between states.
}

Last month, representatives from the US and China met to commission China's Center of Excellence on Nuclear Security (CENS) (http://go.nature.com/uvBDmI), which will provide nuclear security training, a venue for demonstrating new technology and a forum for bilateral and regional best-practice discussions. The event demonstrates the continued importance of international collaboration on nuclear energy issues, as well as the central role played by nuclear scientists in informing matters of nuclear policy.

Most future energy scenarios put stock in nuclear power as a replacement for fossil fuels or to provide baseline for a largely renewable electricity system. Indeed, the International Atomic Energy Agency continues to forecast global growth in nuclear power (http://go.nature.com/K1Na2f). If nuclear energy is going to continue playing a significant and growing role in our energy future, we must deepen our understanding of the risks it poses and build systems around it that prevent its exploitation. Support for nuclear energy research and education should thus be seen as a vital part of continued spending on nuclear energy infrastructure.

Addressing the American Physical Society's March Meeting this year, US Energy Secretary Ernest Moniz affirmed that the physics community will continue to play a critical role in nuclear security. Around the world, organizations like the Center for Energy and Security Studies in Russia or the Center for Science and International Security at the University of Hamburg provide invaluable technical support in ensuring that nuclear power remains as safe as possible from security threats. The CENS will add to that list, strengthening nuclear security and safety throughout China and Asia.

A powerful example of the value of the technical understanding provided by such institutions and by nuclear physicists more generally is the recent Iranian nuclear energy deal. Iran has been working on nuclear technology to varying degrees since the 1970s. Despite being a member of the Treaty on the Non-Proliferation of Nuclear Weapons, Iran's nuclear programme has frequently and consistently raised the spectre of weapons development. Following numerous inspections, UN Security Council Resolutions and mounting international concerns over enrichment, the Joint Plan of Action was drawn up in late 2013 between Iran and the P5+1 (the five permanent members of the UN Security Council China, France, Russia, the UK and the US - plus Germany). This interim measure brought about a freezing of the Iranian nuclear programme and a lessening of some economic sanctions. Negotiations then began on the Joint Comprehensive Plan of Action (JCPOA) between Iran, the P5+1 and the EU. The framework was agreed upon on 14 July 2015 and will span the next 10 to 15 years.

We are now some two months past JCPOA Implementation Day, 18 January 2016, the day on which the plan came into effect. Under the JCPOA, Iran agreed to a number of measures and restrictions on its nuclear power programme - including a reduction of its uranium stockpile and a cap on its enrichment, restrictions on the number and deployment of centrifuges, and a banning of heavy-water reactors - in exchange for the lifting of a broad array of sanctions imposed on it by the UN, the US, and the EU, including an end to the ban on oil imports.

Significant political understanding was required to ensure that a deal could be reached that all parties agreed on - one which would allow Iran to continue its pursuit of nuclear power but which the other nations were confident would not allow them to also pursue nuclear weapons development undetected.

Significant scientific knowledge was required to ensure that this could happen. Crucially, verification - not trust - had to form the cornerstone of the agreement, with robust procedures in place to certify that the atom was being exploited for peace only. Finding technical solutions that could appease all sides was no simple feat, calling on teams of expert nuclear physicists from across the globe.

First, it was important to determine the current nuclear capability of Iran and its likely 'breakout time': the amount of time required to make enough material for a nuclear weapon. There are several ways to make such material, depending on a number of different technological factors. Second, a route was needed that would allow for enrichment of uranium into nuclear fuel but with sufficient constraints to make any proliferation detectable.
Nuclear physicists played a vital role in these assessments and plans. In particular, they were faced with the challenge of redesigning existing nuclear facilities to make them compliant with the technical demands of peaceful operation. This task was complicated by the fact that the Iranian government did not want to scrap parts from its existing investments, imposing significant design constraints on all involved. The IR-40 reactor in Arak, for instance, began construction as a heavy-water reactor. Because of the plutonium-generating capability of that approach, a new design was sought that removed the heavy-water reliance while preserving the partial infrastructure already in place. In the end, the technological restrictions of the JCPOA push Iran's breakout time up to at least a year, essentially removing their capability to build a weapon from their nuclear power programme.

For all of the technical solutions explored and implemented in the JCPOA, it's important to remember the timespan of the current deal. After ten years, the end of the restrictions does not prevent a return to paths that could produce weapons. The JCPOA buys a ten-year window of opportunity in which other plans could be devised to limit enrichment in other Middle Eastern nations embarking on nuclear power programmes. But it also buys time to engage Iran as part of the international community and build trust, particularly with the US.

International scientific collaboration plays a potentially important role here. As in any academic discipline, sharing and engagement between scientists can help to build bridges and foster understanding, while deepening knowledge. This is, however, particularly thorny for nuclear power. Although the international nuclear physics community may be keen to get started, it's still early days in terms of Iran. Moniz is nonetheless hopeful that discussions among scientists can help (http://go.nature.com/gTMVWw).

Through centres like CENS, it is hoped that technical and diplomatic know-how and international collaboration can combine to bolster nuclear security as nuclear power continues to grow. Although reasoned and informed political discourse must find lasting solutions to tensions, knowledge of nuclear physics will undoubtedly continue to play a powerful role over the coming years. 